\title{
Emotional Intelligence and Academic Performance: A Randomized and Gender-Based Multivariate Analysis of Malaysian Undergraduate Students
}

\author{
Rabiu Muazu Musa ${ }^{*}$, Mohamad Razali Abdullah ${ }^{2}$, Ahmad Bisyri Husin Musawi \\ Maliki $^{3}$, Norlaila Azura Kosni ${ }^{4}$, Siti Musliha Mat-Rasid ${ }^{5}$ and Faiza Renaldi ${ }^{6}$ \\ ${ }^{1}$ Centre for Fundamental and Continuing Education, University Malaysia Terengganu, 21030 Kuala Nerus, Terengganu, \\ Malaysia. \\ ${ }^{2}$ East Coast Environmental Research Institute University Sultan Zainal Abidin, 21300 Kuala Nerus, Terengganu, \\ Malaysia. \\ ${ }^{3}$ Faculty of Applied Social Science, University Sultan Zainal Abidin, 21300, Kuala Nerus, Terengganu, Malaysia \\ ${ }^{4}$ Faculty of Sports Science \& Recreation, University Technology Malaysia, Pahang, Malaysia. \\ ${ }^{5}$ Faculty of Sports Science and Coaching, University Education Sultan Idris, Perak, Malaysia. \\ ${ }^{6}$ Informatics Department, Universities Jenderal Achmad Yani, Bandung Barat, Indonesia. \\ *Corresponding author. E-mail: rabiumuazu86@gmail.com
}

\begin{abstract}
Teaching and learning process, particularly in a higher institution of learning, is majorly dependent on student mental preparation which is often viewed as a crucial indicator in predicting effective learning that would, in turn, bring about educational success. The present investigation aims at assessing the Emotional Intelligence (EI) status of undergraduate students and its relationship with the students' academic performance. A total number of 298 students in one of the higher institutions in Malaysia from a myriad discipline were recruited to participate in the study. The EI status of the students, as well as their academic performances, were collected and k-Means clustering was used to group the students in respect to their EI status whilst and Man Whitney test was carried out to ascertain the difference in academic performance and the gender of the students sampled. Two groups were formed from the k-Means clustering method namely, High Emotionally Intelligent Students (HEIST) and Low Emotionally Intelligent Students (LEIST). The Man-Whitney test demonstrated that the HEIST group has higher academic performance as well as better in emotional awareness and management, social-emotional awareness, and relationship management as compared to LEIST $\mathrm{p}<0.05$. Female students were found to be significantly better in relationship management as opposed to males. EI plays a vital role in students' academic performance and therefore, emotional skills management should be highlighted in higher institution of learning to enhance students overall academic performance.
\end{abstract}

Keywords: Emotional Intelligent, Academic Performance, Undergraduate Students, Multivariate Analysis,

Teaching and Learning

\section{INTRODUCTION}

The increase in the research related to emotional intelligence (EI) in the past decade has evident that EI could be assessed reliably and can be applied in predicting essential outcomes, such as managerial efficiency, relationship quality as well as self-development in a multiple dimension. The application of EI intervention is currently receiving a wider recognition and has thus far been demonstrated to be successful in a wide variety of areas including enhancing EI development, health sector, clinical populations as well as school-age settings [1]-[3]. In the aspect of teaching and learning, EI studies have been carried out in view to demonstrate the importance of the concept towards the development of both teachers and students in the learning domain. To this effect, various possibilities of EI application were thus far proposed to enhance the aforementioned domain. 
The applications of the EI assessment tool towards the improvement of classroom activities have received attention from many researchers. A good number of researchers advocated the benefits of teachers' EI in the classroom domain. A study of EI as a predictive indicator in classroom management has been investigated by the preceding researchers [2]. The authors investigated the association between the teacher's EI, the management they adopt in the classroom and how some personal, as well as professional elements, influence teachers' EI. The emotional skills and competence questionnaire for teachers, the teachers, effectiveness scale in classroom management and a personal and professional datasheet were used as the instruments for data collection. The findings from the study revealed that the teachers who possess a higher level of EI are more efficient in managing a classroom. It was concluded from results that EI positively influences the management the teachers make in a classroom and therefore, recommended that training teachers in the aspect of EI are vital in the enhancement of teaching and learning process.

The teacher-student relationship quality and student math achievement has been investigated in prior research [4]. A longitudinal structural equation modeling was applied to investigate the level in which the teacher self-efficacy mediated the teacher-student relation quality as well as math achievement of 881 school children. It was inferred from the study that teacher-student closeness accounted for moderate and large positive effects on the teacher's selfefficacy, conversely, the teacher-student conflict accounted for small moderate negative effects on teacher self-efficacy, which also had small positive effects on math achievement. It was also demonstrated from the findings that closeness could only reflect indirect effects on math achievement with respect to teacher self-efficacy, whereas, conflict revealed a direct and indirect effect on math achievement. It was also reported that the results are consistent across grades. The authors concluded that teacher self-efficacy, as well as student-teacher relationship quality, could potentially enhance student's success in math and as such should be developed.

Carried out a study on how teachers' perceptions of EI, social and emotional learning skills, and teaching efficacy relate to perceptions of teacher-student relationships and students' emotional and behavioral difficulties [5]. A total of 98 teachers from public schools were recruited and completed Self-Rated emotional EI scale, the studentteacher relationships scale, the teacher beliefs scale, and the Strengths and Difficulties Questionnaire (SDQ) for 617 of their students, aged 6-11 years old. A structural equation modeling analysis was utilized to analyze the variables under investigation. The results of the study demonstrated that teachers' perceptions of EI, self-beliefs as well as teaching efficacy were significantly associated with teacher-student relationships, however, not related to students' emotional and behavioral difficulties. Hitherto, the teacher's perceptions of teacher-student relationships were significantly correlated to these difficulties. The author concluded that enhancing teacher's personal and professional skills through EI could foster good teacher- student relationships, prevent relational conflict as well as arrest student's emotional and behavioral difficulties.

The role of English as a foreign language (EFL) Teachers' emotional intelligence towards their teaching success was investigated by the previous researchers [6]. The authors examined the teacher's emotional intelligence, years of teaching experience as well as age and their association with pedagogical success in EFL teaching. A total number of 89 EFL teachers were selected from different language institutes and completed Bar-On's EQ test while the students simultaneously completed a questionnaire for characteristics of EFL teachers who taught the students. Regression analysis demonstrated a significant relationship between teachers' success and EI. A significant positive association between teachers' EI, their teaching experience as well as their age were found. It was concluded from the study that EI is a vital component that promotes successful teaching and learning process.

A study of the effect of EI on job performance of primary school teachers in Malaysia was recently conducted on reff [7] Convenient sampling technique was used to recruit primary school teachers who are teaching in Mata Ayer, Perlis. The EI scale, as well as job performance scale, were used to assess the teachers' level of EI and their overall job performances. The findings from the study revealed that there are associations between the teacher's delivery of performance and the corresponding emotional intelligence. It was concluded from the findings that there exists a relationship between the level of EI, workload as well as job performance amongst primary school teachers.

It could be observed from the previous related works conducted that the application of EI in the teaching and learning domain has received wider recognition. It has been shown from the various findings reported that EI has been successfully used as an intervention technique toward improving teaching and learning as well as promoting teacher's personal quality. However, many of such studies exclusively focused on teachers in either primary or secondary levels of learning. It is worth noting that little or no attention has thus far directed towards the investigation of the EI status of the students at the higher institutions of learning. Consequently, assessing the status of the students EI could their pave a way for the implementation of the necessary intervention that is non-trivial in ensuring a successful academic performance that could ultimately bring about a livelier learning institution as well as competent graduates.

\section{METHODOLOGY}

\subsection{Participants}

The participants of the present study comprised of a total number of 298 undergraduate students with mean aged and standard deviation (19.8 \pm 0.86 years). The students are from University Malaysia Terengganu drawn from various faculties consisting of Economics, Policy management, 
Maritime Management, Accounting, Marketing, Financial mathematics, Food science, Computer science, Biodiversity as well as Chemical science. A total of 84 respondents were male students while 214 students were female. A total of 227 (76.2\%) respondents were Malays, $35(11.8 \%)$ respondents were Chinese, 29 (9.7\%) respondents were Indians and $7(2.3 \%)$ respondents were from other racial groups. Before the commencement of data collection in this study, the students were informed about the purpose of the research and informed consent was obtained.

\subsection{The instrument for Data Collection}

The quick emotional intelligence assessment developed by the preceding investigators will be used as a tool for assessing the emotional intelligence status of the lecturers under study [8]. The instrument measures four fundamental elements of EI constructs; namely, Selfawareness, which is essentially the ability to understand ones 'emotions as they occur. It reflects the learning of where ones' emotions emanate from, the reasons behind them as well as how they are exhibited. Self-management deals with the act of managing one's emotions which consequently mediate reactions and behaviors. This element primarily involves applying self-awareness to emotions which permits self-control that could lead to stronger relations, improved communication and having the opportunity to act rationally and reasonably in a varying situation. Social awareness is the third element measured in the questionnaire, social awareness is the ability to remove focus from oneself and consider other external factors in a view to ascertain their emotions. This involves observing environments and people as well as having the ability to genuinely consider and accept tones and emotions of others in a different circumstance. The fourth construct is relationship management which is the integration of all the aforesaid elements of the EI to allow for positive relationship building as well as maintenance. It reflects the capacity of an individual to carefully combine self-awareness and others in order to craft and mold interactions. It is worth noting that this instrument is considered appropriate for the proposed research due to its relevance to the teaching and learning situations. Moreover, it is non-trivial to highlight that the instrument has been shown to be valid and reliable in measuring teaching and learning-related activity [3],[9],[10].

\subsection{Reliability and Power analysis}

Reliability testing was carried out prior to the start of the proper analysis in the present study. Internal accuracy reliability has been developed in order to analyze the quality of the responses on the instrument items. Cornbrash's alpha coefficient was used to analyze the degree of accuracy between the items and to ensure that the items were analyzed in a single structure (unidimensional) and that the student's answers were independent of each other [11],[12]. It is worth noting that the coefficient values of the items demonstrated a satisfactory value ranging from 0.79 to 0.89 . Moreover, a priori power analysis using $\mathrm{G}^{*}$ Power was conducted to determine the sample necessary to draw a meaningful conclusion in the study. A power analysis of multivariate analysis with a power equivalent to .95 and alpha of .05 suggested that a sample size of 150 respondents would be sufficient to detect a medium effect size of 0.25. Similarly, power analysis for the interpretation of variance with a power equivalent to .80 and an alpha of .05 revealed that a sample size of 200 respondents would be effective to identify a medium effect size of .05 [13]. Therefore, a sample size of 298 deemed sufficient to avoid the problem of Type II error [11].

\subsection{Data Analysis}

Clustering is one of the most common techniques of exploratory data processing that is routinely used to gain intuition on the nature of a dataset. Cluster analysis was reported to be helpful in the detection of subgroups or samples with regards to certain observed parameters. Cluster analysis has been documented to be useful in identifying feature's subgroups or samples based on certain observation [14],[15]. A k-means clustering algorithm is a technique of taxonomy analysis that sequentially tends to split a dataset into a separate kpredefined as well as non-overlapping subgroup known as clusters in which only one group is assigned to each data point. In this step, the algorithm aims to make inter-cluster data points as homogeneous as possible while keeping the intra-clusters as dissimilar as possible. In the present study, the k-means clustering algorithm is applied to group the students based on their CGPA grades as well as the EI scores. The Euclidean distance was used a distance metric of portioning the two clusters formed i.e., High Emotionally Intelligent Students (HEIST) and Low Emotionally Intelligent Students (LEIST). On the other hand, the Mann-Whitney U test was applied to ascertain the differences in investigated variables between the HEIST and the LEIST group.

\section{RESULTS AND DISCUSSION}

The current study was aimed at investigating the relationship between the perceived EI of undergraduate students and their corresponding academic performance. The study also sought to ascertain the differences of EI with respect to the gender of the students. The present study employed a non-conventional approach in assessing the link between the EI status of the students as well as their academic performances. The utilization of multivariate analysis of $\mathrm{k}$-means clustering coupled with the Man Whitney test serve useful in extracting information to ascertain the difference in academic 
performance and the gender of the students sampled. It is worth to mention that the current study is amongst the first study to consider applying a non-conventional technique in evaluating the EI status and academic performance of students. This study is deemed timely due to the relative importance of identifying the psychological elements that could affect learning experience of the students who are often expected to do well in their studies regardless of the challenges pose by the school system as well as a learning process.

Figure 1 shows the grouping of the students with respect to their EI status as well as the CGPA grades. It could be seen from the figure that clear demarcation between the HEIS and the LEIS is provided by the k-means clustering algorithm which further suggests that the group of the students differ in their EI level. This separation enables us to further the analysis by looking at the differences in the EI levels of the HEIS and LEIS via Mann-Whitney U Test.

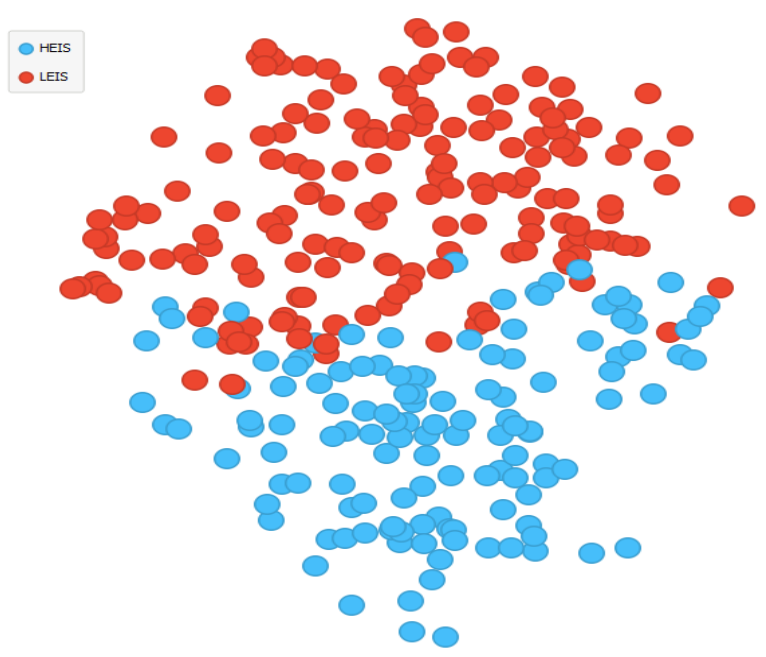

Figure 1: EI Clusters defined by k-means analysis

Table 1: Differences in EI scores and academic performance between the students

\begin{tabular}{llcccc}
\hline Variables & Group & $\mathrm{N}$ & Mean & SD & P(MWU) \\
\hline Emotional & LEIS & 170 & 25.324 & 4.033 & $0.001^{*}$ \\
Awareness & HEIS & 128 & 28.734 & 4.203 & \\
Emotional & LEIS & 170 & 25.412 & 3.506 & $0.001^{*}$ \\
Management & HEIS & 128 & 31.125 & 3.423 & \\
Social- & LEIS & 170 & 28.118 & 4.265 & \multirow{2}{*}{$0.001^{*}$} \\
Emotional & & 128 & 34.297 & 4.187 & \\
Awareness & HEIS & 170 & 23.071 & 3.620 & $0.001^{*}$ \\
Relationship & LEIS & 178 & 28.500 & 3.794 & \\
Management & HEIS & 128 & 3.281 & 0.407 & $0.001^{*}$ \\
CGPA & LEIS & 170 & 3.281 & 0.389 & \\
\hline
\end{tabular}

The findings from the present study in the Man-Whitney test demonstrated that the HEIST group have higher academic performance as well better in emotional awareness and management, social-emotional awareness and relationship management as compared to LEIST $\mathrm{p}<$ 0.001 (Table 1). It has been reported that academic achievement in a higher institution of learning is viewed as the capability of the students to acquire a degree or diploma within a stipulated period as prescribed by the institution [14],[16]. Therefore, it is important to note that the students who can deal with stress as well as cope with the negative thoughts in the mind are found to have a better result as well as improved academically [17],[18].

Table 2: Gender Differences with respect to EI scores and academic performance of the students

\begin{tabular}{llcccc}
\hline Variables & Gender & $\mathrm{N}$ & Mean & SD & $\begin{array}{c}\text { P } \\
\text { MWU) }\end{array}$ \\
\hline Emotional & Male & 84 & 26.821 & 4.407 & 0.989 \\
Awareness & Female & 214 & 26.776 & 4.456 & \\
Emotional & Male & 84 & 27.512 & 5.055 & 0.366 \\
Management & Female & 214 & 28.005 & 4.231 & \\
Social- & Male & 84 & 29.905 & 5.476 & \multirow{2}{*}{$0.022^{*}$} \\
Emotional & & & & & \\
Awareness & Female & 214 & 31.112 & 5.087 & \\
Relationship & Male & 84 & 25.595 & 4.680 & 0.506 \\
Management & Female & 214 & 25.327 & 4.531 & \\
CGPA & Male & 84 & 3.416 & 0.404 & 0.100 \\
& Female & 214 & 3.336 & 0.409 & \\
\hline
\end{tabular}

Table 2 project the differences in the EI scores and the academic performance between male and female students. It could be observed from the table that a significant difference was found between the students in the aspect of social-emotional awareness $\mathrm{p}=0.022$. This result reflects that the female students are better in the aspect social as well as emotional awareness when compared to the male students. It has been inferred that emotional intelligence is a sub-attribute of social intelligence as such in the academic domain, the terms referred to the evaluation as well as the use of emotions in an intelligent way [19],[20]. Moreover, it has been previously documented that social intelligence is the ability of an individual to comprehend and relate appropriately with men, women, boys or girls within a social context [21]. It is worth noting that the importance of applying the appropriate behavior when dealing with different people in the society could translate to the total function functioning and effective interpersonal relationship of an individual that may facilitate the planning as well as the attainment of daily life goals. 


\section{CONCLUSION}

In an attempt to increase student academic success, higher learning institutions have actively involved stakeholders in curriculum modification, the implementation of a new concept and the launch of a myriad support structure to improve academic performance. However, despite these efforts, students do have both satisfactory and unsatisfactory improvement. The present investigation assessed the EI status of undergraduate students and their relationship with the students' academic performance. It has been demonstrated from the findings of the present investigation that EI plays a vital role in students' academic performance and therefore, emotional skills management should be highlighted in Malaysian higher institution of learning to enhance students overall academic performance.

\section{REFERENCES}

[1] E. Lopez-Zafra, M. M. Ramos-Álvarez, K. El Ghoudani, O. Luque-Reca, J. M. Augusto-Landa, B. Zarhbouch, M. Pulido-Martos. Social support and emotional intelligence as protective resources for wellbeing in Moroccan adolescents. Frontiers in psychology ,10 (2019) 1529. DOI : https://doi.org/10.3389/fpsyg.20 19.01529

[2] T. Pozo-Rico, R. Gilar-Corbí, A. Izquierdo, J. L. Castejón. Teacher Training Can Make a Difference: Tools to Overcome the Impact of COVID-19 on Primary Schools. An Experimental Study. International Journal of Environmental Research and Public Health ,17 (22) (2020) 8633. DOI : https://doi.org/10.3390/ijer ph17228633

[3] S. M. Moon. Book Review: Emotional development and emotional intelligence: Educational implications . Gifted Child Quarterly, 43 (2) (1999) 108-110. DOI : https://doi.org/10.1177/001698629904300208

[4] D. B. Hajovsky, K. A. Oyen, S. R. Chesnut, S. J. Curtin. Teacher-student relationship quality and math achievement: The mediating role of teacher self efficacy. Psychology in the Schools, 57 (1) (2020) 111134. DOI : https://doi.org/10.1002/pits.22322

[5] M. S. Poulou. An examination of the relationship among teachers' perceptions of social-emotional learning, teaching efficacy, teacher-student interactions, and students' behavioral difficulties. International Journal of School \& Educational Psychology, 5 (2) (2017) 126-136. DOI : https://doi.org/10.1080/2168360 3.2016.1203851
[6] A. Ghanizadeh, F. Moafian. The role of EFL teachers' emotional intelligence in their success. ELT journal, 64 (4) (2010) 424-435. DOI : https://doi.org/10 $.1093 / \mathrm{elt} / \mathrm{ccp} 084$

[7] M. Mohamad, J. Jais. Emotional intelligence and job performance: A study among Malaysian teachers .Procedia Economics and Finance, 35 (2016) 674-682. DOI : https://doi.org/10.1016/S2212-5671(16)00083-6

[8] J. M. Dewaele, K. V. Petrides, A. Furnham. Effects of trait emotional intelligence and sociobiographical variables on communicative anxiety and foreign language anxiety among adult multilinguals: A review and empirical investigation. Language learning, 58 (4) (2008) 911-960. DOI : https://doi.org/10.1111/j.14679922.2008.00482.x

[9] A. K. Vesely, D. H. Saklofske, A. D. Leschied. Teachers-The vital resource: The contribution of emotional intelligence to teacher efficacy and wellbeing. Canadian Journal of School Psychology, 28 (1) (2013) 71-89. DOI : https://doi.org/10.1177/082957351 2468855

[10] N. De Bondt, P. Van Petegem. Emphasis on emotions in student learning: Analyzing relationships between overexcitabilities and the learning approach using Bayesian MIMIC modeling. High Ability Studies ,28 (2) (2017) 225-248. DOI : https://doi.org/10.1080/1 3598139.2017.1292897

[11] R. M. Musa, J. A. Kannan, U. K. W. Nek, N. W. F. M. Rashid, A. M. Ali, N. A. S. A. Rashid. Appraisal of wellness-lifestyle status among Malaysian undergrad uate students: A cross-sectional and gender-based wellness survey. Journal of Applied Pharmaceutical Science, 10 (09) (2020) 082-087. DOI : 10.7324/JAPS. 2020.10910

[12] J. E. Helms, K. T. Henze, T. L. Sass, V. A. Mifsud. Treating Cronbach's alpha reliability coefficie nts as data in counseling research. The counseling psyc hologist, 34 (5) (2006) 630-660. DOI : https://doi.org/ $10.1177 / 0011000006288308$

[13] J. Cohen. A power primer. Psychological bulletin, 112 (1) (1992) 155. DOI : https://doi.org/10.1 037/0033-2909.112.1.155 
[14] R. M. Musa, M. Z. Suhaimi, A. Musa, M. R. Abdullah, A. P. P. Abdul Majeed, A. B. H. M. Maliki. Predicting students academic performance from wellness status markers using machine learning techniques. Indian Journal of Science and Technology ,13 (29) (2020) 2047-2055. DOI : https://doi.org/ 10.17 485/IJST/v13i29.999

[15] R. M. Musa, M. Haque. Academic performance of pre-clinical and clinical medical students' of east coast Malaysian peninsula: a cross-sectional and descriptive study that stimulates their life. Journal of Applied Pharmaceutical Science, 7 (06) (2017) 169-175. DOI : 10.7324/JAPS.2017.70624

[16] B. H. Chew, A. M. Zain, F. Hassan. Emotional intelligence and academic performance in first and final year medical students: a cross-sectional study. BMC medical education, 13 (1) (2013) 1-10.

[17] R. C. L. Chao. Managing stress and maintaining well being: Social support, problem focused coping, and avoidant coping. Journal of Counseling \& Develo pment, 89 (3) (2011) 338-348. DOI : https://doi.org/10. 1002/j.1556-6678.2011.tb00098.x

[18] M. Richardson, C. Abraham, R. Bond. Psychological correlates of university students' academic performance: a systematic review and metaanalysis. Psychological bulletin, 138 (2) (2012) 353.

[19] M. J. Fambrough, R. Kaye Hart. Emotions in leadership development: A critique of emotional intelligence. Advances in Developing Human Resourc es, 10 (5) (2008) 740-758. DOI : https://doi.org/10.1177 $/ 1523422308323542$

[20] L. Bunce, A. J. Lonsdale, N. King, J. Childs, R. Bennie. Emotional intelligence and self-determined behaviour reduce psychological distress: Interactions with resilience in social work students in the UK. The British Journal of Social Work, 49 (8) (2019) 20922111. DOI : https://doi.org/10.1093/bjsw/bcz008

[21] M. A. Brackett, S. E. Rivers, S. Shiffman, N. Lerner, P. Salovey. Relating emotional abilities to social functioning: a comparison of self-report and performance measures of emotional intelligence .Journal of personality and social psychology, 91 (4) (2006) 780. DOI : https://doi.org/10.1037/0022-3514.9 1.4.780 